\title{
App CovidIDOSOS um aplicativo para auxiliar no tratamento de idosos com COVID-19
}

\author{
Ana Maria Silva Paiva ${ }^{1}$, Alisson Clementino da Silva ${ }^{1}$, Cecir Barbosa de Almeida \\ Farias $^{1}$, João Vitor da Silva Alves ${ }^{1}$, Pedro Florêncio Almeida Lima ${ }^{1}$ \\ ${ }^{1}$ Universidade Federal de Campina Grande (UFCG) \\ Rua Luiz Grande, S/N, Frei Damião, CEP 58540-000 - Sumé - PB - Brazil \\ a mpaiva@outlook.com, aliclementesilva@gmail.com, \\ cecir.barbosa@professor.ufcg.edu.br, joaovitors.alves8@gmail.com, \\ pedrocgfal95egmail.com
}

\begin{abstract}
The application described by this article is being developed for use by health professionals and the elderly with the test positive for Covid-19, acting with support because in many cases there is the aggravating factor of preexisting diseases causing the need for treatment assistance. The application aims to offer information and support to health professionals, in addition to educational and healthy actions in the daily lives of elderly patients with Covid19, for help them about the importance of prevention, medical treatment and care after contamination of this disease.
\end{abstract}

Resumo. O aplicativo COVIDOSOS descrito por este artigo está sendo desenvolvido para uso de profissionais da saúde e idosos que testaram positivo para o Covid-19, atuando com suporte, pois em muitos casos há o fator agravante de doenças pré-existentes causando a necessidade de auxílio no tratamento. O aplicativo visa oferecer informações e suporte aos profissionais da saúde, além de ações educacionais e saudáveis o cotidiano dos pacientes idosos portadores de Covid-19, a fim de despertá-los para a importância da prevenção, do tratamento médico e cuidados após a contaminação desta doença.

\section{Introdução}

Na época de epidemia da Covid-19, surge a necessidade de novos enfoques tecnológicos que auxiliem aos pacientes, familiares, agentes de saúde, enfermeiros e médicos durante o tratamento. A tecnologia pode torna-se uma aliada neste momento difícil possibilitando uma assistência e análise completa, podendo também observar a evolução do quadro clínico, principalmente tratando de pacientes idosos. O envelhecimento, ainda que saudável, torna nosso sistema imunológico mais frágil e, por isso, há que se redobrar os cuidados. Por isto, uma série de características intrínsecas tornam esse grupo mais vulnerável à Covid-19.

A luta contra o novo coronavírus não envolve apenas coragem, mas racionalidade, paciência e ciência, deste modo, o cuidado para idosos aliado a tecnologia pode torna-se uma grande ferramenta neste momento difícil possibilitando uma assistência e análise completa, podendo também observar a evolução do quadro clínico. Por isto, o aplicativo em questão surge como um suporte estendido do hospital, seguindo as necessidades dos pacientes e profissionais da saúde. 


\section{Fundamentação Teórica}

A infecção pelo n-CoV-2019, causa uma série de complicações com o principalmente com o aumento da idade. O risco de contagio se desenvolver para morte, aumenta com a idade, já que a imunossenescência aumenta a perda da capacidade do corpo para responder a infecções imunológica e infectocontagiosas, tornando pessoas de terceira idade uma grande parcela dos óbitos globais (HUANG et al, 2020).

Alguns trabalhos vem sendo realizados para auxiliar no tratamento da Covid-19, tais como, no Brasil, o Ministério da Saúde lançou o aplicativo "Coronavírus-SUS" com o objetivo de conscientizar a população sobre o Corona Vírus, com as funcionalidades: os sintomas, como se prevenir, o que fazer em caso de suspeita e infecção; mapa indicando unidades de saúde próximas e em caso de suspeita de infecção, o cidadão será instruído para a unidade de saúde básica mais próxima (CORONAVÍRUS SUS, 2020).

Outra ferramenta digital é um "bot” no Telegram que conversa com o profissional e disponibiliza boletins diários da secretaria de Saúde pelo Brasil e o número de casos pelo Brasil. Os médicos também podem acompanhar o panorama geral da Covid-19 através de uma "dashboard" atualizada em tempo real com informações sobre os casos em todo o Brasil. Os médicos brasileiros podem utilizar também um aplicativo com fluxos interativos, manejos e tratamentos da Covid-19, de acordo com notícias da Sanar Medicina (2020).

Foi percebido que nenhum dos trabalhos encontrados proporciona o tratamento para pacientes com Covid-19 e comunicação com equipe médico-hospitalar como o aplicativo aqui proposto (COVIDOSOS).

\section{Materiais e Métodos}

O aplicativo COVIDOSOS foi desenvolvido utilizando a ferramenta Mit App Inventor, utilizando a linguagem de programação em blocos lógicos e o Cloud $D B$, para armazenamento em nuvem. Este aplicativo possui interfaces e layouts amigáveis para idosos e é também uma ferramenta prática para médicos, enfermeiros, e agentes de saúde pois estes podem buscar dados e informações dos pacientes e acompanhar o tratamento.

O aplicativo está sendo desenvolvido por uma equipe de estudantes e professora da UFCG em um projeto de extensão vinculado ao PROBEX - Programa de Bolsas de Extensão, e a partir de reuniões com equipe do projeto, médicos, enfermeiras e agentes de saúde, foram criados (na etapa de análise) diagramas, tais como: o Diagrama de Use Case (Figura 1). O diagrama demonstra um cenário de todas as funcionalidades do sistema, com as funções de cadastrar usuários, apresentar informações, editar e remover dados de usuários e prescrições. Observa-se que os médicos e enfermeiros possuem funcionalidades diferentes dos agentes de saúde e dos pacientes.

\section{Resultados}

A tela inicial do aplicativo é apresentada na Figura 2, assim como também, é apresentada a tela seguinte para acesso ao aplicativo, através da digitação de dados de login. Na tela de login o usuário pode solicitar um novo cadastro ou realizar login. Para realizar o login 
dos pacientes é necessário digitar o número do cartão do SUS e para profissionais é necessário o CPF. Em ambos os casos, a senha contém os números da data de nascimento.

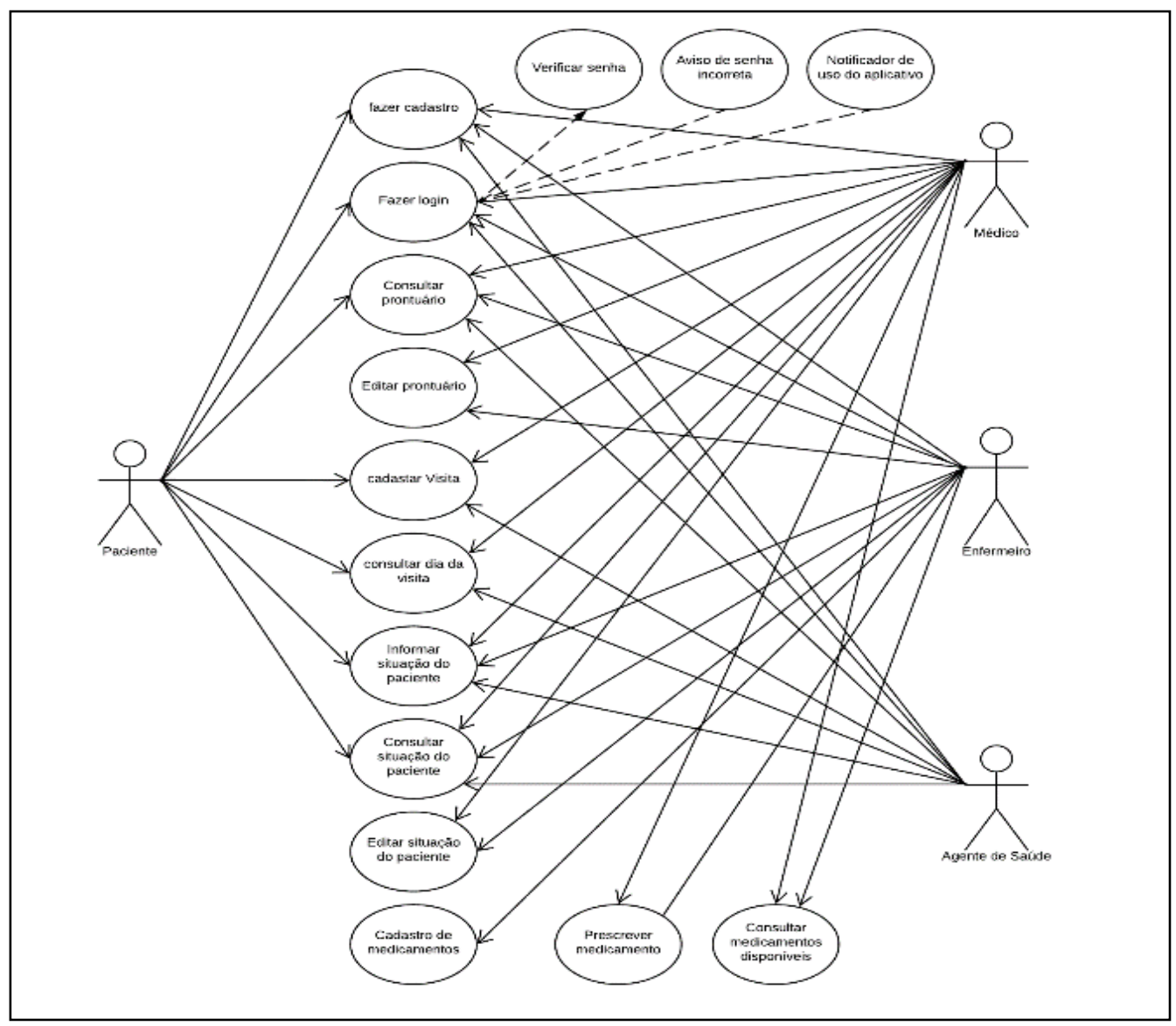

Figura 1. Diagrama de Use Case

O aplicativo COVIDOSOS proporciona aos pacientes idosos, portadores de Covid-19 e outras doenças crônicas, a obtenção de acesso a informações importantes do seu tratamento (Figura 2), pois cada usuário possui uma tela de menu com botões que permitem acessar funções específicas, e possibilita que eles saibam mais sobre seus tratamentos, como por exemplo: horários das medicações prescritas pelos médicos (tanto para sintomas do coronavirus, como também medicações para suas doenças crônicas préexistentes), e caso eles estejam período de quarentena em casa, poderão obter informações sobre visitas de agentes de saúde. Os pacientes também possuirão possibilidade de comunicação com os profissionais de saúde através do aplicativo, e poderão enviar dados para sobre sintomas da doença que possuem a cada dia.

Este aplicativo funciona como um bom instrumento para os profissionais da área de saúde, pois oferece suporte com muitas funcionalidades possibilitando assim que os profissionais obtenham mais controle sobre os pacientes idosos contaminados, como por exemplo: acesso a ficha do paciente e situação de cada um, com visualização de dados das últimas atualizações, a partir do número do cartão do SUS do paciente. Os profissionais podem inserir dados de novos pacientes, prescrição de plano terapêutico, prontuário, editar e visualizar dados de boletim do paciente, visitas, ou verificar a situação 
atual de cada paciente, além de cadastrar, atualizar ou excluir medicamentos e unidades de saúde (Figura 2).
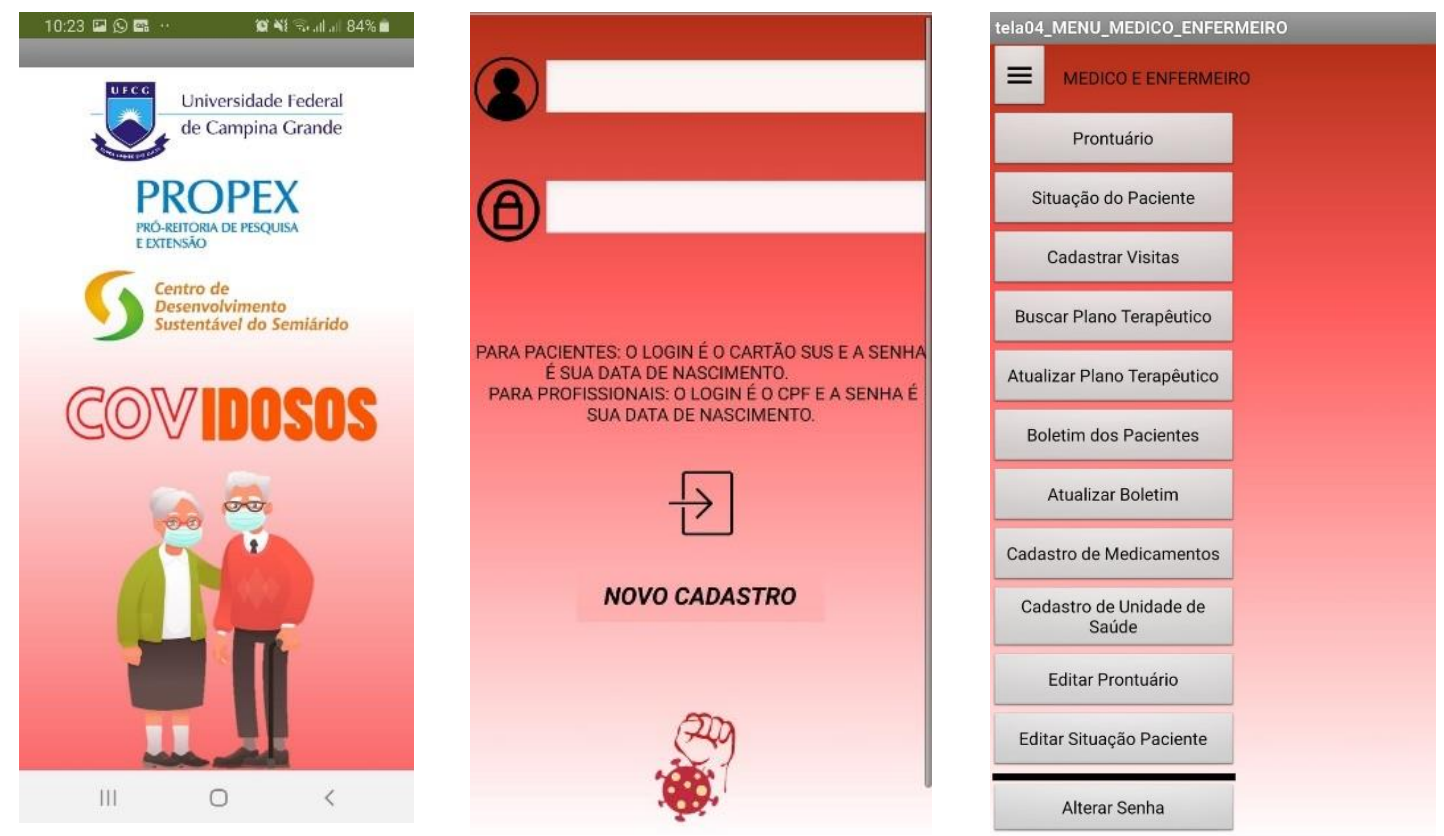

Figuras 2. A) Tela Inicial do aplicativo e B) tela de Login C) tela de menus

$\mathrm{Na}$ sua primeira versão, o aplicativo realiza sincronização com os dados informados por todos os usuários a partir de dados armazenados na nuvem (Cloud $D B$ ). Foi realizada uma série de testes com os usuários do hospital e algumas novas funcionalidades precisarão ser desenvolvidas, tais como: a emissão de relatórios e gráficos estatísticos.

\section{Conclusão}

O aplicativo desenvolvido atendeu aos objetivos definidos inicialmente, possibilitando ao usuário monitoramento e avaliação de pacientes em tratamento da Covid-19. O aplicativo possibilita o cadastro de unidades de saúde, pacientes, profissionais, medicamentos e prescrições a partir de consultas clínicas (ou visitas presenciais a domicílio), plano terapêutico e boletim de paciente, facilitando o tratamento, atendendo a pacientes, médicos, enfermeiros e agentes de saúde. O aplicativo será disponibilizado na Play Store e proporcionará eficiência no monitoramento e avaliação dos pacientes, diminuindo os abandonos e possibilitando avanço na cura da doença. O aplicativo também poderá ser utilizado em Hospitais e unidades de saúde semelhantes.

\section{Referências}

CORONAVÍRUS SUS. Governo do Brasil. Abril, 2020.

HUANG, Chaolin; WANG, Yeming; LI, Xingwang; et al. Clinical features of patients infected with 2019 novel coronavirus in Wuhan, China. The Lancet, 2020.

MIT App Inventor Disponível em: <https://appinventor.mit.edu/> 2012. Sanar Medicina. Cinco ferramentas grátis contra o Coronavírus para médicos. Maio, 2020. 